\title{
Electron-Beam Manipulated Nanoscale Reaction
}

Huaping Sheng ${ }^{1,2}$, Jianbo Wang ${ }^{2,3, *}$, Jianguo Wen ${ }^{1, *}$

1. Center for Nanoscale Materials, Argonne National Laboratory, Lemont, IL, USA

2. School of Physics and Technology, Center for Electron Microscopy, MOE Key Laboratory of

Artificial Micro- and Nano-structures, and Institute for Advanced Studies, Wuhan University, Wuhan, China

3. Science and Technology on High Strength Structural Materials Laboratory, Central South

University, Changsha, China

* Corresponding author: wang@whu.edu.cn; jwen@anl.gov

Employing electron beam for nanofabrication is highly desirable since it provides both the highest resolution and location flexibility. Sun et al. has demonstrated that reversible oxidation and reduction of Ag in a gas cell containing air could be controlled by changing the e-beam current density in TEM [1]. However, the gas cell contains high concentration of oxygen such that the redox reaction is extremely intense. In this work, taking advantage of low oxygen partial pressure in TEM, we show direct visualization of the redox of $\mathrm{Ag}$ at atomic resolution and manipulation of redox reaction at nanoscale.

A thin foil of $\mathrm{Ag}$ was ion milled to electron transparency for the TEM experiment. No noticeable oxidization is observed on the surface of thin area, after leaving the TEM specimen inside microscope overnight. As shown in Fig. 1, a nucleation of $\mathrm{Ag}_{2} \mathrm{O}$ island is observed on the surface of $\mathrm{Ag}$, similar to

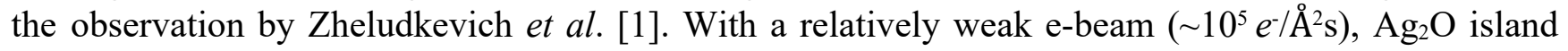
continues to grow, with crystallographic orientation relationship: $\left\langle 110>_{\mathrm{Ag}} / /<110>_{\mathrm{Ag}_{2} \mathrm{O}}\right.$ and $\{111\}_{\mathrm{Ag}} / /$ $\{002\}_{\mathrm{Ag}_{2} \mathrm{O}}$. When increasing beam intensity to a dose rate of $\sim 10^{6} e^{-/} \AA^{2} \mathrm{~s}, \mathrm{Ag}_{2} \mathrm{O}$ begins to shrink,

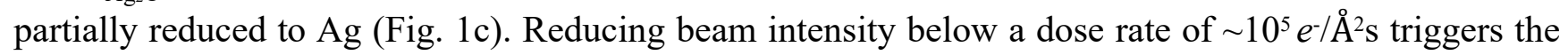
growth of $\mathrm{Ag}_{2} \mathrm{O}$ again as shown in Fig. 1d. The reversible redox of $\mathrm{Ag}$ is achieved by varying the e-beam dose rate and irradiation time.

It is highly desirable to directly control nanoscale reaction using electron beam due to its potential utility in the fabrication of nanoscale devices. By reducing electron beam size to few nanometers with suitable dose rate, we demonstrated fabrication of an $3 \times 3$ array of $3 \mathrm{~nm} \mathrm{Ag} 2 \mathrm{O}$ nanodots in an $\mathrm{Ag}$ matrix. As shown in Fig. $2 \mathrm{a}$, some of nanodots such as dots \#2, \#5, \#6, \#7, \#9 are fully converted into $\mathrm{Ag}_{2} \mathrm{O}$. Enlarged \#5 nanodots in Fig. $2 \mathrm{~b}$ clearly shows the lattice difference between $\mathrm{Ag}_{2} \mathrm{O}$ and the $\mathrm{Ag}$ matrix. Fast Fourie Transformation (FFT) pattern in Fig. 2c also supports the conclusion. However, some nanodots like dots $\# 1$ and $\# 4$ are not converted into $\mathrm{Ag}_{2} \mathrm{O}$ yet and some of them like \#3 and \#8 are drilled into a hole. Using through focus imaging, we found out there exists a small amount of $\mathrm{Ag}_{2} \mathrm{O}$ exists on surface. Due to the large thickness at $\# 1$ and $\# 4$, these two nanodots are partially fully converted into $\mathrm{Ag}_{2} \mathrm{O}$ nanodots. As for nanodots $\# 3$ and $\# 8$, a small amount $\operatorname{lof}_{2} \mathrm{Ag}$ at the edge of the drilled nanodots is observed, indicating that $\mathrm{Ag}_{2} \mathrm{O}$ nanodots were formed and then drilled into a hole due to over-time irradiation. Therefore, a dynamic monitor of the reaction status and an automatic stopping beam system is needed for complete manipulation of nanoscale reaction. We plan to carry out high-speed imaging using a high-speed direct electron detector; real-time on-the-fly data processing with a capability for the phase formation; and immediate feedback to the microscope to make smart decisions including choosing beam location, diameter, intensity, dwelling time, etc. [3] 
References:

[1] ML Zheludkevich et al., Oxid. Met. 61 (2004), p. 39.

[2] L Sun et al., Langmuir 27 (2011), p. 14201.

[3] Electron microscopy was performed in the Center for Nanoscale Materials, a U.S. Department of Energy Office of Science User Facility under Contract No. DE-AC02-06CH11357. H. Sheng and J. Wang acknowledge the financial support from the National Natural Science Foundation of China (51671148, 51271134, J1210061, 11674251, 51501132, 51601132), the Hubei Provincial Natural Science Foundation of China (2016CFB446, 2016CFB155), the Fundamental Research Funds for the Central Universities, and the CERS-1-26 (CERS-China Equipment and Education Resources System), and the China Postdoctoral Science Foundation (2014T70734), and the Open Research Fund of Science and Technology on High Strength Structural Materials Laboratory (Central South University) and the Suzhou Science and Technology project (No. SYG201619).

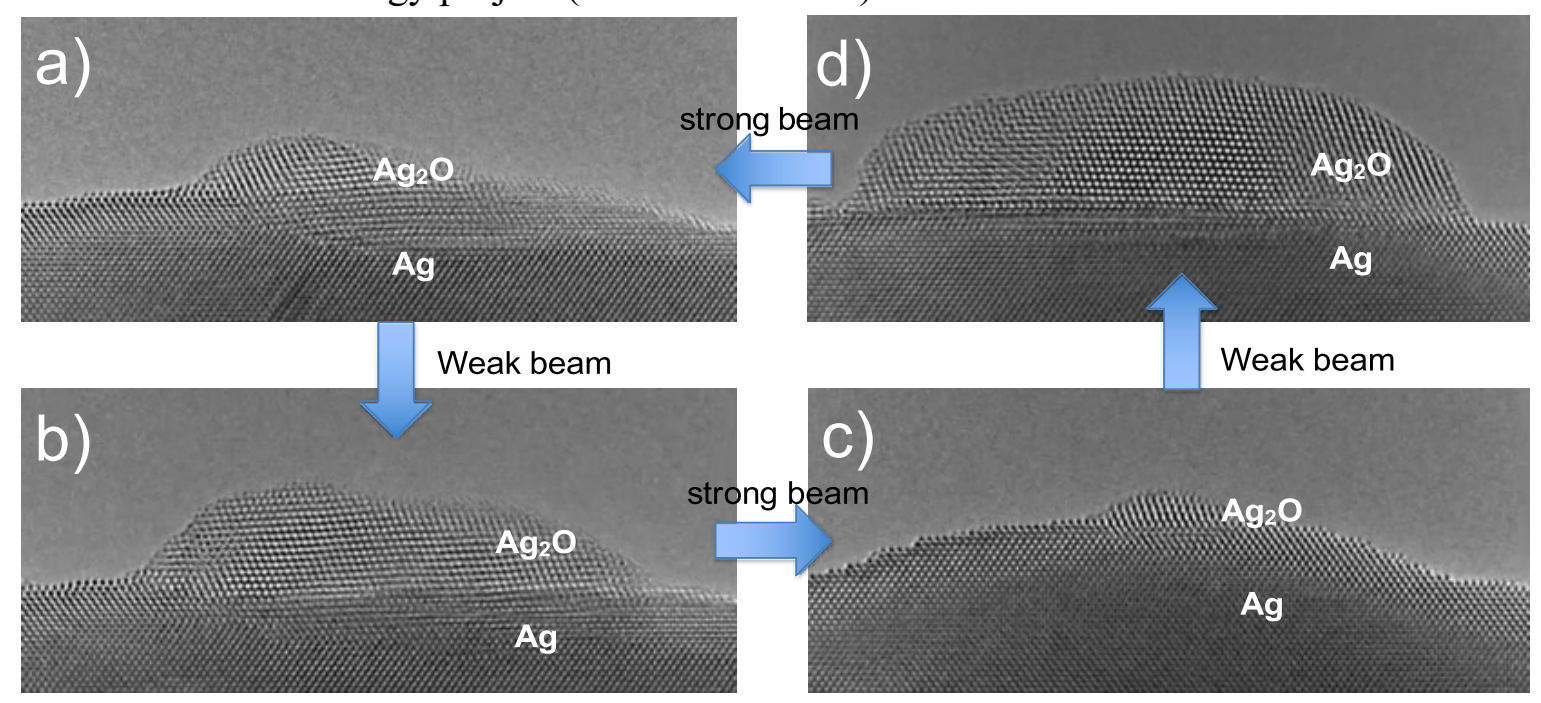

Figure 1. HREM images of redox of Ag by varying e-beam intensity. a) initial growth of Ag2O island; b) further growth of $\mathrm{Ag}_{2} \mathrm{O}$ under weak beam $\left(\sim 10^{5} e^{-/} \AA^{2} \mathrm{~s}\right)$; c) reduction of $\mathrm{Ag}_{2} \mathrm{O}$ into $\mathrm{Ag}$ under strong beam $\left(\sim 10^{6} e^{\left.-/ \AA^{2} s\right)}\right.$; d) regrowth of $\mathrm{Ag}$ under weak beam.

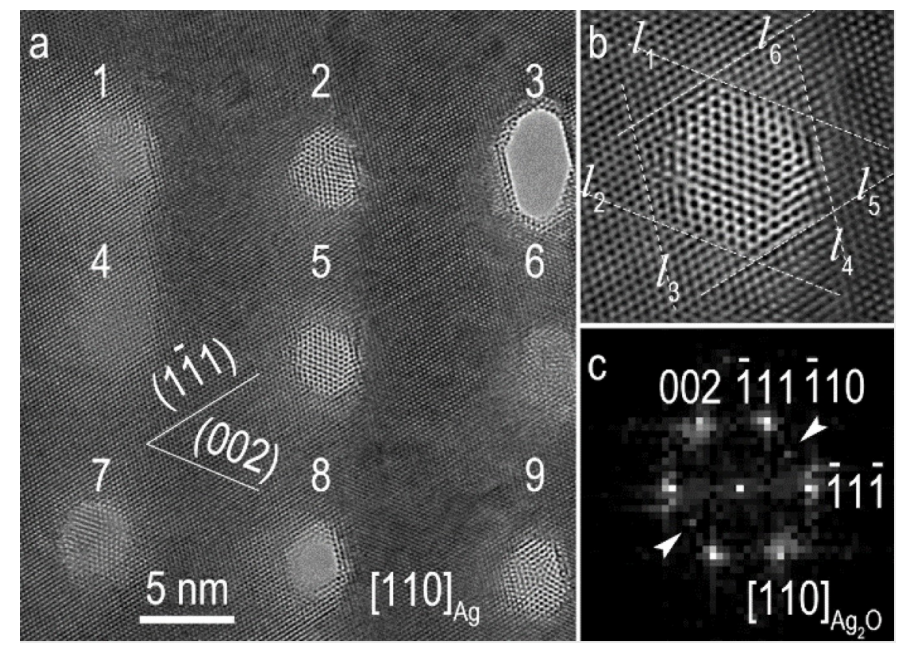

Figure 2. Manipulation e-beam to form $\mathrm{Ag}_{2} \mathrm{O}$ nanodots in $\mathrm{Ag}$ matrix. 